PROCEEDINGS OF THE

AMERICAN MATHEMATICAL SOCIETY

Volume 126, Number 9, September 1998, Pages 2635-2640

S 0002-9939(98)04366- 4

\title{
HYPERGROUPS WITH INVARIANT METRIC
}

\author{
MICHAEL VOIT
}

(Communicated by Palle E. T. Jorgensen)

\begin{abstract}
The purpose of this note is to extend the following classical result from groups to hypergroups in the sense of C.F. Dunkl, R.I. Jewett, and R. Spector: If a hypergroup has a countable neighborhood base of its identity, then $K$ admits a left- or a right-invariant metric. Moreover, it admits an invariant metric if and only if there exists a countable conjugation-invariant neighborhood base of the identity.
\end{abstract}

A classical result due to Birkhoff [1], Kakutani [5] and others states that each second countable locally compact group $G$ admits a left-invariant metric $d$, i.e., one has

$$
d(x, y)=d(z x, z y) \quad \text { for all } x, y, z \in G .
$$

The purpose of this note is to prove a result of this kind for hypergroups in the sense of Dunkl, Jewett, and Spector. In this case the convolution of two points $x, y$ is a compactum $\{x\} *\{y\}$ and usually no longer a single point. In this case one usually finds elements $x, y$ in the hypergroup with $x \neq y$ and $(\{z\} *\{x\}) \cap(\{z\} *\{y\}) \neq \emptyset$. This shows that one cannot expect to obtain an invariant metric $d$ satisfying a strong invariance property like $d(x, y)=d(a, b)$ for all $x, y, z, a, b \in K$ with $a \in\{z\} *\{x\}$ and $b \in\{z\} *\{y\}$. However, the following weaker condition can be achieved:

Definition 1. Let $K$ be a hypergroup with identity $e$ such that the topology of $K$ is generated by some metric $d$. Then $d$ is called left-invariant, if for all $x \in K$ and $\epsilon>0$ the $\epsilon$-balls $U_{\epsilon}(x):=\{y \in K: d(x, y)<\epsilon\}$ satisfy $U_{\epsilon}(x)=\{x\} * U_{\epsilon}(e)$.

Right-invariance is defined in the same way, and $d$ is called invariant if it is both left- and right-invariant.

If the hypergroup is a group $G$, then it can easily seen that our left-invariance just means that $d(e, y)=d(z, z y)$ for all $z, y \in G$. As this implies that

$$
d(x, y)=d\left(e, x^{-1} y\right)=d\left(z x,(z x)\left(x^{-1} y\right)\right)=d(z x, z y) \quad \text { for all } x, y, z \in G,
$$

it follows that for groups our definition agrees with the classical one stated in Eq. (1).

The following theorem is the main result of this note:

Theorem 1. If a hypergroup has a countable neighborhood base of its identity, then it admits a right-invariant metric.

Received by the editors December 26, 1996 and, in revised form, January 27, 1997.

1991 Mathematics Subject Classification. Primary 43A62; Secondary 20N20, 54E35.

Key words and phrases. Hypergroups, invariant metric.

(C)1998 American Mathematical Society 
Assume that a hypergroup $K$ admits an invariant metric $d$. Then all $\epsilon$-balls $U_{\epsilon}(e)$ around $e$ satisfy $\{x\} * U_{\epsilon}(e)=U_{\epsilon}(e) *\{x\}$ for all $\epsilon>0$ and $x \in K$, which means that there exists a countable "conjugation-invariant" neighborhood base of the identity $e \in K$. Therefore, one direction of the following theorem is clear:

Theorem 2. A hypergroup admits an invariant metric if and only if there exists a countable conjugation-invariant neighborhood base of its identity.

Remarks. (1) If a commutative hypergroup $K$ has a countable neighborhood base of its identity, then these neighborhoods trivially are conjugation-invariant, and $K$ admits an invariant metric.

(2) For any discrete hypergroup, the metric $d$ with $d(x, y)=1$ for $x \neq y$ is invariant.

(3) We presently do not know whether each second countable compact hypergroup admits a conjugation-invariant neighborhood base of $e$ and hence an invariant metric.

Before we prove the theorems, we recapitulate some notation and facts about hypergroups; for further details see [2] and [4].

Definition 2. A hypergroup $(K, *)$ consists of a locally compact Hausdorff space $K$ together with a bilinear, associative, weakly continuous convolution on the Banach space $M_{b}(K)$ of all bounded regular Borel measures on $K$ with the following properties:

(1) For all $x, y \in K$, the convolution of the point measures $\delta_{x} * \delta_{y}$ is a probability measure with compact support.

(2) The mapping $K \times K \longrightarrow \mathcal{C}(K),(x, y) \longmapsto \operatorname{supp}\left(\delta_{x} * \delta_{y}\right)$, is continuous with respect to the Michael topology on the space $\mathcal{C}(K)$ of all nonvoid compact subsets of $K$, where this topology is generated by the sets

$$
U_{V, W}:=\{L \in \mathcal{C}(K): L \cap V \neq \emptyset, L \subset W\} \quad \text { with } V, W \text { open in } K .
$$

(3) There is an identity $e \in K$ with $\delta_{x} * \delta_{e}=\delta_{e} * \delta_{x}=\delta_{x}$ for all $x \in K$.

(4) There is a continuous involution $x \mapsto \bar{x}$ on $K$ such that $\left(\delta_{x} * \delta_{y}\right)^{-}=\delta_{\bar{y}} * \delta_{\bar{x}}$ and $e \in \operatorname{supp}\left(\delta_{x} * \delta_{y}\right) \Longleftrightarrow x=\bar{y}$ for $x, y \in K$.

We use the common abbreviation $A * B:=\bigcup_{x \in A, y \in B} \operatorname{supp}\left(\delta_{x} * \delta_{y}\right)$ for sets $A, B \subset K$. Moreover, $\bar{A}$ is the image of $A \subset K$ under the involution on $K$. The closure of $A$ is denoted by $\mathrm{cl} A$.

The following facts from Sections 2.5, 3.2, and 4.1 of Jewett [4] will be needed in the proof of the theorems:

(A) For all $A, B, C \subset K$, we have $(A * B) \cap C=\emptyset \quad \Longleftrightarrow A \cap(C * \bar{B})=\emptyset$.

(B) If $U$ is a neighborhood of the identity $e$ in $K$, then for all $A \subset K, \operatorname{cl} A \subset A * U$.

(C) For all compacta $A, B \subset K$ with $A \cap B=\emptyset$ there exists a neighborhood $U$ of $e$ with $(A * U) \cap(B * U)=\emptyset$.

Proof of Theorem 1. The proof will be divided into three major steps.

Step 1. Assume the hypergroup $K$ has a countable neighborhood base of its identity $e$. For $k \in \mathbb{N}$ we choose inductively a neighborhood base $\left(U_{k}\right)_{k \in \mathbb{N}}$ of $e$ consisting of open neighborhoods $U_{k}$ of $e$ with the following properties:

(a) $\bar{U}_{k}=U_{k}$ and $U_{k+1} * U_{k+1} \subset U_{k}$ for all $k$. 
(b) For all $1 \leq n<k$ and all integers $1 \leq l(1)<l(2)<\ldots l(n)<k$,

$$
U_{k} * U_{l(1)} * U_{l(2)} * \ldots * U_{l(n)} \subset U_{l(1)} * U_{l(2)} * \ldots * U_{l(n)} * U_{k-1} .
$$

In fact, (a) can be achieved obviously for each sufficiently small symmetric $U_{k}$. Moreover, as $\operatorname{cl}\left(U_{l(1)} * U_{l(2)} * \ldots * U_{l(n)}\right) \subset U_{l(1)} * U_{l(2)} * \ldots * U_{l(n)} * U_{k-1}$ by fact (B) above, we conclude from fact (C) above that the finitly many conditions in (b) are satisfied for each sufficiently small $U_{k}$.

As $\left(U_{k}\right)_{k \in \mathbb{N}}$ is a neighborhood base of $e$, we in particular have $\bigcap_{k} U_{k}=\{e\}$. In the next step, we define $V_{2^{-k}}:=U_{k}$ for $k \in \mathbb{N}$. For arbitrary dyadic rational numbers

$$
r:=2^{-l(1)}+2^{-l(2)}+\ldots+2^{-l(n)} \quad \text { with } \quad 0<l(1)<l(2)<\ldots<l(n)
$$

we define

$$
V_{r}:=V_{2^{-l(1)}} * V_{2^{-l(2)}} * \ldots * V_{2^{-l(n)}} \quad \text { for } 0<r<1 .
$$

Moreover, for $r \geq 1$ we put $V_{r}=K$. The sets $V_{r}$ are open, and we next prove that they have the following properties for arbitrary dyadic rational numbers $r, s \geq 0$ :

(1.1) $V_{r} \subset V_{s}$ for $r<s$.

(1.2) $V_{r} * V_{s} \subset V_{r+s+2 \cdot \min (r, s)}$.

Our proof of (1.1) follows the exposition of the proof of the same statement in the proof of Theorem 8.2 of Hewitt and Ross [3]. In order to check (1.1), we may assume $0<r<s<1$. Let $r$ be given as in Eq. (2) and $s$ by $2^{-m(1)}+2^{-m(2)}+\ldots+2^{-m(p)}$ with $0<m(1)<\ldots<m(p)$. Then there is a unique $k$ with $l(j)=m(j)$ for $j<k$ and $l(k)>m(k)$. Letting $W:=V_{2^{-l(1)}} * V_{2^{-l(2)}} * \ldots * V_{2^{-l(k-1)}}$, we then have

$$
\begin{aligned}
V_{r} & =W * V_{2^{-l(k)}} * \ldots * V_{2^{-l(n)}} \subset W * V_{2^{-l(k)}} * V_{2^{-l(k)-1}} * \ldots * V_{2^{-l(n)}} * V_{2^{-l(n)}} \\
& \subset \ldots \subset W * V_{2^{-l(k)+1}} \subset W * V_{2^{-m(k)}} \subset V_{2^{-m(1)}} * V_{2^{-m(2)}} * \ldots * V_{2^{-m(p)}}=V_{s} .
\end{aligned}
$$

In order to prove (1.2), we first assume $0<s \leq r$ with $r+3 s<1$ (notice that the case $r+3 s \geq 1$ is obvious). It suffices here to check (1.2) for $r$ as in (2), and for $s=2^{-l}$ with $l \geq l(1)$. If $l>l(n)$, then we have

$$
V_{r} * V_{2^{-l}}=V_{r+2^{-l}}
$$

and (1.2) holds. If $l \leq l(n)$, then we take $k \in \mathbb{N}$ with $k \geq 2$ and $l(k-1) \leq l<l(k)$. Let $r_{1}:=2^{-l+1}-2^{-l(k)}-2^{-l(k+1)}-\ldots-2^{-l(n)}$ and $r_{2}=r+r_{1}$. Then $r<r_{2}<$ $r+2^{-l+1}$. Applying (1.1) and Eq.(3), we obtain

$$
V_{r} * V_{2^{-l}} \subset V_{r_{2}} * V_{2^{-l}}=V_{r_{2}+2^{-l}} \subset V_{r+2^{-l+1}+2^{-l}}=V_{r+3 \cdot 2^{-l}} .
$$

We next prove that $V_{s} * V_{r} \subset V_{r+3 s}$ for $0<s \leq r$ with $r$ as in Eq.(2) where we again may assume that $s=2^{-l}$ with $l \geq l(1)$. Take again $k$ with $l(k-1) \leq l<l(k)$. Then, by property (b) of the sets $U_{k}$,

$$
\begin{aligned}
V_{2^{-l}} * V_{r} & =V_{2^{-l}} * V_{2^{-l(1)}} * \ldots * V_{2^{-l(k)}} * \ldots * V_{2^{-l(n)}} \\
& \subset V_{2^{-l(1)}} * \ldots * V_{2^{-l(k-1)}} * V_{2^{-l+1}} * V_{2^{-l(k)}} * \ldots * V_{2^{-l(n)}} \subset V_{r+2 \cdot 2^{-l}}
\end{aligned}
$$

which completes the proof of step (1.2).

Step 2. We next define the mappings

$$
\tilde{\phi}(x):=\inf \left\{r: x \in V_{r}\right\}^{1 / 2} \quad \text { and } \quad \phi(x):=\tilde{\phi}(x)+\tilde{\phi}(\bar{x}) \quad \text { for } x \in K \text {. }
$$

The function $\phi$ has the following properties:

(2.1) $\phi(x)=0$ if and only if $x=e$, and $\phi(x)=\phi(\bar{x})$ for all $x \in K$. 
(2.2) For all $a, b \in K$ and $c \in\{a\} *\{b\}$, one has $\phi(c) \leq \phi(a)+\phi(b)$.

(2.3) The sets $W_{\epsilon}(e):=\{x \in K: \phi(x)<\epsilon\}$ form a neighborhood base of $e$ for $\epsilon>0$, and, in particular, $\phi$ is continuous at $e \in K$.

The symmetry of $\phi$ in (2.1) is clear. Moreover, $\phi(x)=0$ means that $x, \bar{x} \in V_{r}$ for all dyadic $r>0$, which is equivalent to the fact that $x, \bar{x} \in U_{k}$ for all $k \in \mathbb{N}$, and this means that $x=e$. Hence, part (2.1) is clear.

In order to check (2.2), take $\epsilon>0$. We then find dyadic numbers $r_{1}, r_{2}>0$ with $a \in V_{r_{1}}, b \in V_{r_{2}}, \tilde{\phi}(a)^{2}+\epsilon>r_{1}$, and $\tilde{\phi}(b)^{2}+\epsilon>r_{2}$. Then by (1.2),

$$
c \in\{a\} *\{b\} \subset V_{r_{1}} * V_{r_{2}} \subset V_{r_{1}+r_{2}+\min \left(r_{1}, r_{2}\right)}
$$

which implies that

$$
\tilde{\phi}(c)^{2} \leq r_{1}+r_{2}+2 \min \left(r_{1}, r_{2}\right) \leq \tilde{\phi}(a)^{2}+\tilde{\phi}(b)^{2}+2 \min \left(\tilde{\phi}(a)^{2}, \tilde{\phi}(b)^{2}\right)+4 \epsilon .
$$

As this holds for all $\epsilon>0$, we conclude that

$$
\tilde{\phi}(c)^{2} \leq \tilde{\phi}(a)^{2}+\tilde{\phi}(b)^{2}+2 \min \left(\tilde{\phi}(a)^{2}, \tilde{\phi}(b)^{2}\right) \leq(\tilde{\phi}(a)+\tilde{\phi}(b))^{2} .
$$

It is now obvious from the definition of $\phi$ that (2.2) holds.

In order to check (2.3), we first notice that for $\epsilon>0$ and dyadic numbers $r$ with $0<r<\epsilon / 2$, the set $V_{r} \cap \bar{V}_{r}$ is an open neighborhood of $e$ with $V_{r} \cap \bar{V}_{r} \subset W_{\epsilon}(e)$, which shows that all $W_{\epsilon}(e)$ are neighborhoods of $e$. Conversely, for each open neighborhood $U$ of $e$ we find $U_{k}$ with $U_{k} \subset U$. Therefore, $W_{2^{-k}} \subset U_{k} \subset U$, and (2.3) becomes clear.

Step 3. We define the metric $d$ on $K$ by

$$
d(x, y):=\inf \{\phi(z): z \in\{x\} *\{\bar{y}\}\} \quad \text { for } x, y \in K .
$$

We still have to prove that $d$ has the required properties:

(3.1) It is clear that for all $x, y \in K, d(x, y) \geq 0$ and $d(x, x)=0$. Moreover, if $x \neq y$, then $e$ is not contained in the compactum $\{x\} *\{\bar{y}\}$. By (2.3) this yields that there exists $\epsilon>0$ with $W_{\epsilon}(e) \cap(\{x\} *\{\bar{y}\})=\emptyset$, and hence $d(x, y) \geq \epsilon>0$.

(3.2) $d$ is symmetric, as $\{x\} *\{\bar{y}\}=(\{y\} *\{\bar{x}\})^{-}$and as $\phi$ is symmetric by (2.1).

(3.3) To check the triangle inequality, take $x, y, z \in K$ and $\epsilon>0$. We then find $a \in\{x\} *\{\bar{y}\}$ and $b \in\{y\} *\{\bar{z}\}$ with $\phi(a) \leq d(x, y)+\epsilon$ and $\phi(b) \leq d(y, z)+\epsilon$. Thus, $x \in\{a\} *\{y\}$ and $y \in\{b\} *\{z\}$, and $x \in\{a\} *\{b\} *\{z\}$. This implies that $(\{a\} *\{b\}) \cap(\{x\} *\{\bar{z}\}) \neq \emptyset$. Now choose some $c \in(\{a\} *\{b\}) \cap(\{x\} *\{\bar{z}\})$. The definition of $d$ together with (2.2) ensures that

$$
d(x, z) \leq \phi(c) \leq \phi(a)+\phi(b) \leq d(x, y)+d(y, z)+2 \epsilon .
$$

The triangle inequality now follows for $\epsilon \rightarrow 0$, and we now know that $d$ is a metric.

(3.4) In order to prove the right-invariance of $d$, choose $\epsilon>0$ and $x \in K$ and define

$$
W_{\epsilon}(x):=\{z \in K: d(x, z)<\epsilon\} .
$$

Then this notion is consistent with that of (2.3) for $x=e$. Moreover, for each $y \in W_{\epsilon}(e) *\{x\}$ we find $z \in K$ with $d(z, e)<\epsilon$ and $y \in\{z\} *\{x\}$, which yields $z \in\{y\} *\{\bar{x}\}$, and hence, by the definition of $d, d(x, y) \leq d(z, e)<\epsilon$. This implies that

$$
W_{\epsilon}(e) *\{x\} \subset W_{\epsilon}(x)
$$


Conversely, if $y \in W_{\epsilon}(x)$, then $d(x, y)<\epsilon$, and we find $z \in\{y\} *\{\bar{x}\}$ with $\phi(z)=d(e, z)<\epsilon$ and thus $z \in W_{\epsilon}(e)$. Thus, $y \in\{z\} *\{x\} \subset W_{\epsilon}(e) *\{x\}$, and the converse inclusion is also proved.

(3.5) We still have to check that $\rho$ generates the original topology. This, however, immediately follows from the fact that the following three assertions are equivalent for all $x, z_{n} \in K(n \in \mathbb{N})$ :

(a) $\lim _{n \rightarrow \infty} z_{n}=x$ with respect to the original topology.

(b) For $\epsilon>0$ there is $n_{0}$ with $\left(\{x\} *\left\{\bar{z}_{n}\right\}\right) \cap W_{\epsilon}(e) \neq \emptyset$ for $n \geq n_{0}$.

(c) For $\epsilon>0$ there is $n_{0}$ with $z_{n} \in W_{\epsilon}(x)$ for $n \geq n_{0}$.

In fact, the equivalence $(\mathrm{a}) \Longleftrightarrow$ (b) follows from the continuity axiom (2) of hypergroups above while $(\mathrm{b}) \Longleftrightarrow$ (c) is a consequence of (3.4).

The proof of Theorem 1 is now complete.

Proof of Theorem 2. The proof will again be divided into three steps, which are similar to, but sometimes easier than, the steps in the proof of Theorem 1.

Step 4. Choose a neighborhood base $\left(U_{k}\right)_{k \geq 1}$ of $e$ such that for all $k \in \mathbb{N}$,

(a) $\bar{U}_{k}=U_{k}$ and $U_{k+1} * U_{k+1} \subset U_{k}$, and

(b) $U_{k}$ is conjugation-invariant.

The symmetry and the conjugation-invariance of the $U_{k}$ ensures that the sets $V_{r}$ as defined in step 1 of the proof of Theorem 1 have the following stronger properties for all dyadic rational numbers $r, s \geq 0$ :

(4.1) $V_{r} \subset V_{s}$ for $r<s$,

(4.2) $V_{r} * V_{s} \subset V_{r+s}$,

(4.3) $V_{r}$ is conjugation-invariant and symmetric.

Step 5. The function

$$
\phi(x):=\inf \left\{r: x \in V_{r}\right\} \quad \text { for } x \in K
$$

has the following properties:

(5.1) $\phi(x)=0$ if and only if $x=e$,

(5.2) $\phi(\bar{x})=\phi(x)$ for all $x \in K$,

(5.3) The sets $W_{\epsilon}(e):=\{x \in K: \phi(x)<\epsilon\}$ form a neighborhood base of $e$ for $\epsilon>0$.

(5.4) All $W_{\epsilon}(e)$ are conjugation invariant.

In fact, it suffices to check (5.4): Take $y \in\{x\} * W_{\epsilon}(e)$. Then there is $z \in K$ with $y \in\{x\} *\{z\}$ and $\phi(z)<\epsilon$. We find a dyadic rational $r<\epsilon$ with $z \in V_{r}$. Hence, by the conjugation invariance of $V_{r}, y \in\{x\} *\{z\} \subset\{x\} * V_{r}=V_{r} *\{x\} \subset W_{\epsilon} *\{x\}$, which proves $\{x\} * W_{\epsilon}(e) \subset W_{\epsilon} *\{x\}$. The converse inclusion can be checked in the same way.

Step 6. This step can be carried out in the same way as in the previous theorem where the additional left-invariance of $d$ follows from (5.4). This completes the proof of Theorem 2 .

Examples. Hypergroup structures on intervals $I \subset \mathbb{R}$ were studied and partially classified by Zeuner [6]. He proved that on $\mathbb{R}$ each hypergroup structure is isomorphic with the group $(\mathbb{R},+)$. He also showed that each hypergroup structure on 
$[0, \infty[$ or $[0,1]$ is commutative and can be normalized (after a suitable isomorphism) as follows:

(1) If $K=[0, \infty[$, then $|x-y|, x+y \in\{x\} *\{y\} \subset[|x-y|, x+y]$ for all $x, y \in[0, \infty[$.

(2) If $K=[0,1]$, then $|x-y|, x+y \in\{x\} *\{y\} \subset[|x-y|, x+y]$ for all $x, y \in[0,1]$ with $x+y \leq 1$.

It can easily be checked that the usual metric on $[0, \infty[$ or $[0,1]$ is invariant for these hypergroups.

\section{REFERENCES}

1. G. Birkhoff: A note on topological groups. Compos. Math. 3, $427-430$ (1936).

2. W.R. Bloom, H. Heyer: Harmonic Analysis of Probability Measures on Hypergroups. De Gruyter (1995). MR 96a:43001

3. E. Hewitt, K.A. Ross: Abstract Harmonic Analysis I. Springer-Verlag: Berlin - New York (1979). MR 81k:43001

4. R.I. Jewett: Spaces with an abstract convolution of measures. Adv. Math. 18, $1-101$ (1975). MR 52:14840

5. S. Kakutani: Über die Metrisation der topologischen Gruppen. Proc. Imp. Acad. Tokyo 12, 82 -84 (1936).

6. Hm. Zeuner: One-dimensional hypergroups. Adv. Math. 76, 1 - 18 (1989). MR 90i:43002

Mathematisches Institut, Universität Tübingen, 72076 Tübingen, Germany

E-mail address: voit@uni-tuebingen.de

Department of Mathematics, University of Virginia, Kerchof Hall, Charlottesville, VIRGINIA 22903-3199 